\title{
interview
}

\section{A new twist for communications}

\author{
Twisted light beams with different values of orbital angular momentum can be used to provide dramatic \\ increases in the capacity of free-space optical communications. Nature Photonics spoke to Alan Willner at \\ the University of Southern California to find out more.
}

\begin{abstract}
Where did you get the idea of using orbital angular momentum as a means of multiplexing for communications?

The existence of orbital angular momentum (OAM) has been known for a long time. In the early 1990s, Leslie Allen and his colleagues at Leiden University were the first to link Laguerre-Gaussian modes with the OAM of a photon. In 2001, Anton Zeilinger and co-workers at the University of Vienna made a great step forward when they showed that the entanglement of OAM states could be used for quantum communications. The initial concept of using OAM for classical communications was demonstrated in 2004 by Miles Padgett and co-workers at the University of Glasgow. It was really at that point that the wider community began thinking about using OAM for communications. Another important driving force is due to the 'Information in a Photon' programme, which was started in December 2009 by Mark Neifeld at the Defense Sciences Office of DARPA.
\end{abstract}

\section{What are benefits of using OAM for multiplexing?}

The key point is that OAM provides an additional orthogonal degree of freedom for increasing the capacity of an optical link. OAM is also compatible with polarization- and wavelengthmultiplexing. Although the modal patterns of different OAM beams do overlap, their inherent orthogonality allows them to be demultiplexed efficiently. An important metric for many types of communications is spectral efficiency, which is measured in bits per second per hertz. Because all of these different OAM beams can be located at the same wavelength, you're simply increasing the capacity and efficiency of the channel while not consuming any more bandwidth in the spectral domain. We demonstrated transmission efficiencies of around $25 \mathrm{bit} \mathrm{s}^{-1} \mathrm{~Hz}^{-1}$ in a single group of OAM rings and $95 \mathrm{bit} \mathrm{s}^{-1} \mathrm{~Hz}^{-1}$ for two groups of concentric OAM rings, with each group transmitting more than 1 Tbit s$^{-1}$ through free space on a single wavelength. Extending that to multiple wavelengths would represent a significant advance in terms of capacity.

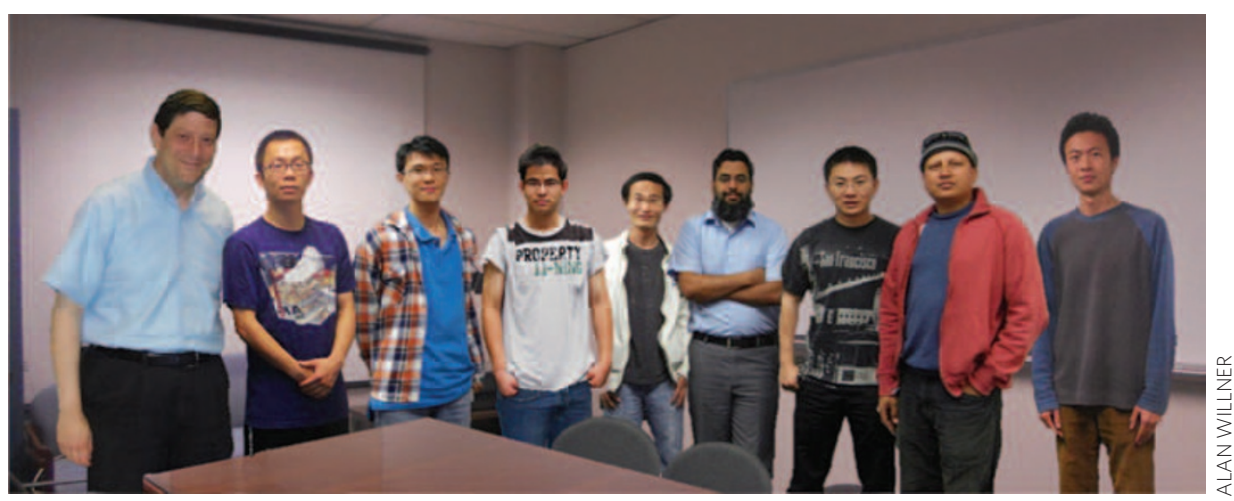

Left to right: Willner, Huang, Yan, Ren, Yang, Ahmed, Wang, Fazal and Yue (Dolinar and Tur absent). The researchers successfully multiplexed beams with different OAM values and showed that data can be swapped between the channels.

How many OAM beams can be multiplexed?

In general, wavelength multiplexing is limited by the size of the available spectral domain, whereas spatial multiplexing is limited by the aperture size of the key elements in the link. Polarization multiplexing, in contrast, provides only two orthogonal channels. For OAM, an infinitely large aperture would theoretically accommodate an infinite number of beams, which would make the scheme similar to wavelength multiplexing in terms of scalability. Of course, crosstalk between modes is a key limitation and therefore must be minimized. We started with four OAM beams and then doubled the data-carrying capacity of each beam three times by employing plus-and-minus OAM charges, polarization multiplexing and two concentric groups of OAM rings. We envision that at least another order of magnitude increase could be achieved in the future. Schemes for the generation, multiplexing and demultiplexing of OAM beams using superior spatial light modulators or integrated devices would help to improve the maximum number of available OAM beams. We will certainly need a convenient, efficient and scalable way of controlling both amplitude and phase.

What are the prospects for transmission over longer distances in free space?

This is the main goal. One of the challenges in this respect is turbulence in the atmosphere. For situations that require high capacity or spectral efficiency over relatively short distances of less than $1 \mathrm{~km}$, this approach could be appealing. Of course, there are also opportunities for long-distance satellite-to-satellite communications in space, where turbulence is not an issue. Moreover, simulations have shown that a feedback-loop-controlled phase pattern can partially correct for distortions due to turbulence, and we've already used forwarderror correction with OAM beams to improve system performance.

\section{What about transmission over fibre?} Using multiplexed OAM modes over fibre is another exciting prospect. For example, Siddharth Ramachandran and co-workers at Boston University have demonstrated stable OAM mode propagation over $1 \mathrm{~km}$ of a specially designed ring fibre, and Yoshinari Awaji at the National Institute of Information and Communications Technology in Japan has found that multicore fibres in a ring shape can also support OAM modes. But there are still many questions yet to be answered. For example, what is the impact of bending or polarization mode dispersion, and how scalable are these fibre approaches?

\section{INTERVIEW BY OLIVER GRAYDON}

Alan Willner and co-workers have an Article on orbital angular momentum multiplexing on page 488 of this issue. 